\title{
Corticotropin-Releasing Factor Acting at the Locus Coeruleus Disrupts Thalamic and Cortical Sensory-Evoked Responses
}

\author{
David M Devilbiss*,', Barry D Waterhouse ${ }^{2}$, Craig W Berridge' and Rita Valentino ${ }^{3}$ \\ 'Department of Psychology, University of Wisconsin-Madison, Madison, WI, USA; ${ }^{2}$ Department of Neurobiology and Anatomy, Drexel \\ University, Philadelphia, PA, USA; ${ }^{3}$ Department of Anesthesiology and Critical Care Medicine, Children's Hospital of Philadelphia, Philadelphia, \\ PA, USA
}

\begin{abstract}
Stress and stress-related psychiatric disorders, including post-traumatic stress disorder, are associated with disruptions in sensory information processing. The neuropeptide, corticotropin-releasing factor (CRF), coordinates the physiological and behavioral responses to stress, in part, by activating the locus coeruleus-norepinephrine (LC-NE) projection system. Although the LC-NE system is an important modulator of sensory information processing, to date, the consequences of CRF activation of this system on sensory signal processing are poorly understood. The current study examined the dose-dependent actions of CRF at the LC on spontaneous and sensory-evoked discharge of neurons within the thalamus and cortex of the vibrissa somatosensory system in the awake, freely moving rat. Peri-LC infusions of CRF resulted in a dose-dependent suppression of sensory-evoked discharge in ventral posterior medial thalamic and barrel field cortical neurons. A concurrent increase in spontaneous activity was observed. This latter action is generally not found with iontophoretic application of NE to target neurons or stimulation of the LC-NE pathway. Net decreases in signal-to-noise of sensory-evoked responses within both regions suggest that under conditions associated with CRF release at the LC, including stress, the transfer of afferent information within sensory systems is impaired. Acutely, a suppression of certain types of sensory information may represent an adaptive response to an immediate unexpected stressor. Persistence of such effects could contribute to abnormalities of information processing seen in sensorimotor gating associated with stress and stress-related psychopathology.
\end{abstract}

Neuropsychopharmacology (2012) 37, 2020-2030; doi:I0.1038/npp.2012.50; published online I8 April 20I2

Keywords: coeruleus [ceruleus]; cortex (somatosensory); corticotropin-releasing factor (CRF); norepinephrine [noradrenaline]; thalamus (somatosensory)

\section{INTRODUCTION}

Acute stress disrupts sensory information processing (Clark et al, 1986; Ermutlu et al, 2005; Grillon and Davis, 1997) and impairs a variety of cognitive processes, including sustained attention and working memory. These impairments likely contribute to stress-related work place accidents and over 1.9 million stress-related disabling injuries a year (BarriosChoplin et al, 1998). Moreover, deficits in sensory information processing are found in patients with stressrelated psychiatric diseases, particularly post-traumatic stress disorder (PTSD; Orr et al, 2002). These deficits have been best characterized using behavioral tests of prepulse

*Correspondence: Dr DM Devilbiss, Department of Psychology, University of Wisconsin-Madison, 1202 W. Johnson Street, W. J. Brogden Psychology Building Room 523, Madison, WI 53706, USA, Tel: + I 608265 8722, Fax: + I 608262 4029, E-mail: ddevilbiss@wisc.edu Received 3 January 2012; revised 6 March 2012; accepted 19 March 2012 inhibition (PPI; Braff et al, 2001). In this task, both stress and stress-related psychopathology are associated with impairment in the degree to which a weak stimulus can inhibit a subsequent startle response elicited by intense stimuli and is an index of sensorimotor gating (Swerdlow et al, 2001). Although the majority of research on the neurobiology of PPI has focused on the role of limbic circuitry, event-related potential studies demonstrate that sensory information processing of both the pre-stimulus and startle stimulus are deficient in stress and PTSD (Ermutlu et al, 2005; Holstein et al, 2010; Miyazato et al, 2000; Orr et al, 2002), suggesting that stress may directly modulate sensory system function. Currently, our understanding of the neural mechanisms involved in stressrelated changes in sensory signal processing is limited.

Corticotropin-releasing factor (CRF) has a critical role in coordinating the physiological and behavioral responses to stressors (Bale and Vale, 2004; Hauger et al, 2009). In addition to its role as a neurohormone in the median eminence, CRF functions as a neuromodulator to regulate 
central neural, cognitive, and behavioral aspects of stress (Owens and Nemeroff, 1991). The locus coeruleus-norepinephrine (LC-NE) pathway is an important target of the neuromodulatory CRF system (Valentino and Van Bockstaele, 2008; Van Bockstaele et al, 1996). CRF increases tonic firing rates of individual LC neurons (Curtis et al, 1997; Valentino and Foote, 1988), elevating NE release throughout the forebrain (Curtis et al, 1997; Page and Abercrombie, 1999). Stressor-induced increases in LC-NE neurotransmis-

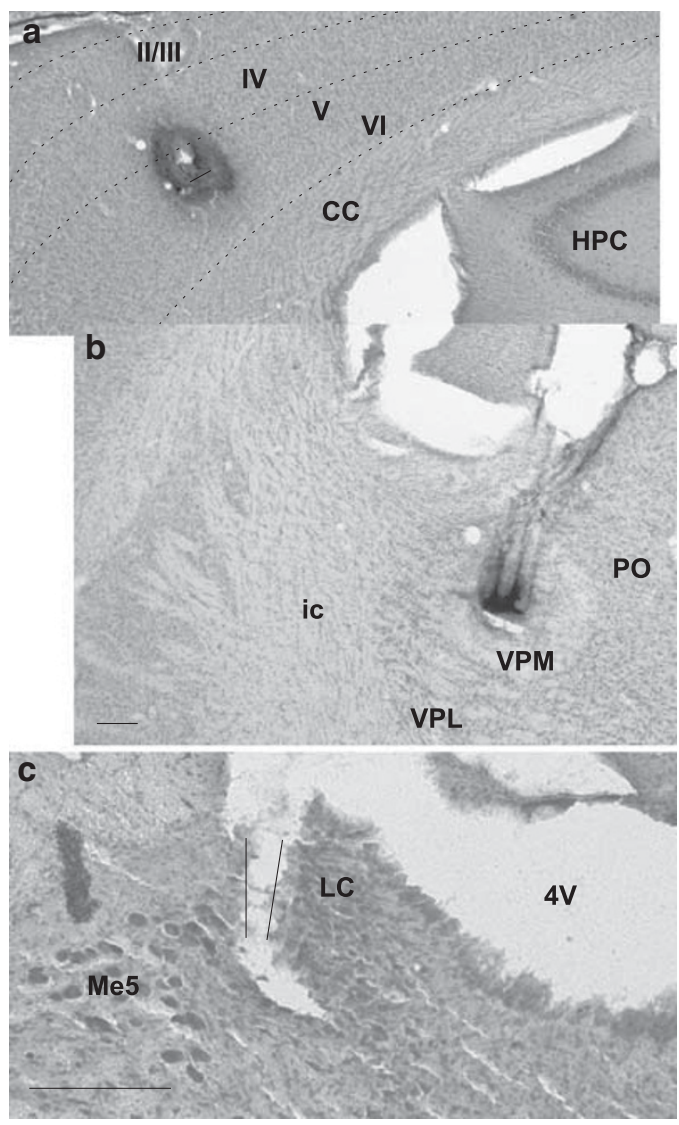

d

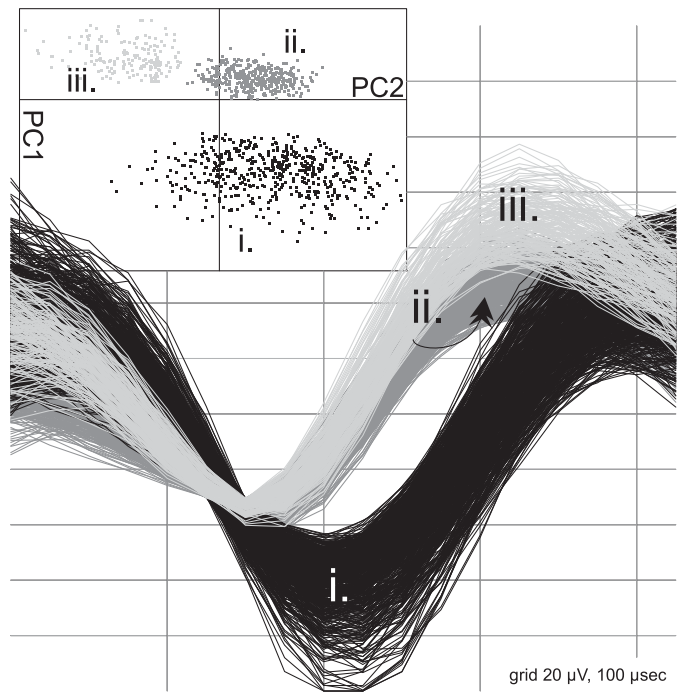

sion are attenuated by administration of CRF antagonists within the LC, suggesting a critical role for CRF in stressrelated activation of the LC (Kawahara et al, 2000; Page et al, 1993; Valentino et al, 1991). Moreover, activation of the LC-NE system suppresses PPI and evoked responsepotential measures of sensory gating, similar to that observed in stress and PTSD (Alsene and Bakshi, 2011; Conti et al, 2002; Ermutlu et al, 2005; Gresack and Risbrough, 2010; Miyazato et al, 2000). Together, these observations suggest that CRF activation of the LC-NE pathway during stress and stress-related disorders likely alters sensory system function.

The neuromodulatory actions of the LC-NE system on target neuron function have been extensively studied using the rat vibrissa (whisker) trigeminal system. Somatosensory information is transmitted by neurons within specialized cytoarchitectonic units of the ventral posteriomedial (VPm) thalamus (ie, 'barreloids') to similar specialized units in the barrelfield (BF) subdivision of the primary somatosensory cortex (ie, 'barrels'; Van der Loos, 1976; Simons, 1978; Waite, 1973). Moreover, the entire rat trigeminal pathway is innervated by LC neuron collaterals (Simpson et al, 1997). Direct activation of the LC-NE pathway or iontophoretic application of NE can facilitate sensory-evoked discharge of individual neurons and neural ensembles within sensory systems according to an inverted-U-shaped neuromodulatory profile (Devilbiss and Waterhouse, 2004; Devilbiss et al, 2006). However, to date, the effects of CRF activation of the LC-NE pathway on sensory system function are unknown.

The current study tested whether CRF acts at the LC to modulate single neuron response properties within the somatosensory system using combined peri-LC infusions of CRF and electrophysiological recordings of individual neurons of the VPm thalamus and BF cortex in awake, freely moving rats. We found that CRF actions at the LC resulted in a suppression of somatosensory signal transmission and increased spontaneous activity of both thalamic and cortical neurons. These results suggest that during stress, CRF acts at the LC to suppress neuronal processing of weak afferent stimuli within sensory systems. This action could contribute to disruptions in sensory information processing and deficient sensorimotor gating in PTSD and other psychiatric disorders.

Figure I Example photomicrographs of the final electrode and infusion locations. (a) $\times 40$ photomicrograph of the barrelfield (BF) cortex with a Prussian blue reaction product indicating the final location of the recording electrode placed in layer $V$ (Black Bar). (b) $\times 40$ photomicrograph somatosensory thalamus with a Prussian blue reaction product indicating the final location of three neighboring recording electrodes placed in the vibrissae VPm subdivision of the thalamus. Electrodes are separated by $>100 \mu \mathrm{m}$. Bar $=200 \mu \mathrm{m}$ (c) $\times 100$ photomicrograph of the pons illustrating the track of the infusion needle lateral to the LC. Bar $=200 \mu \mathrm{m}$. (I-VI, cortical lamina; CC, corpus callosum; HPC, hippocampus; ic, internal capsule; VPL, ventral posterolateral; VPm, ventral posteromedial; PO, posterior thalamic group; Me5, mesencephalic trigeminal nucleus; LC, locus coeruleus; and $4 \mathrm{~V}$, fourth ventricle). (d) Recorded electrical activity from a single microwire electrode positioned in the BF cortex was discriminated as three 'unit' waveforms (i-iii) and represented in a scatter plot (PCI vs PC2; inset). Using our offline criteria, units $\mathrm{i}$-iii were verified as originating from three different single neurons. These neurons were separable in PCA space, though their action potential waveforms overlapped. As such, all three units could be classified as individual neurons. 


\section{MATERIALS AND METHODS}

\section{Animals}

Five adult male Long-Evans hooded rats (Charles River Laboratories, Wilmington, MA) weighing 250-450 g were housed in a temperature- and humidity-controlled environment with ad libitum food and water. Testing was conducted between 1100 and 1500 hours (ie, lights on 0700-1900 hours). All procedures were done in accordance with the NIH guidelines on research and animal care and were approved by the appropriate Institutional Animal Care and Use Committees.

\section{Animals and Surgical Procedures}

Procedures were as described previously (Devilbiss and Waterhouse, 2004). Blunt-tip $50 \mu \mathrm{m}$ stainless-steel Tefloncoated microwires were chronically implanted in layer $\mathrm{V}$ of the C3 whisker representation in BF somatosensory cortex and the corresponding ipsilateral VPm thalamus (Figure 1). A stimulation electrode was threaded under the skin and anchored at the base of the central C3 whisker in the whisker pad. Additionally, a guide cannula (26 gauge) was implanted above the LC-lateral dendritic field for peri-LC infusions. In two animals, a second cannula was placed further lateral from the LC to serve as an anatomical site control (900-1000 $\mu \mathrm{m}$ from the LC nucleus).

Animals were anesthetized with a mixture of $390 \mathrm{mg} / \mathrm{kg}$ chloral hydrate (Sigma, St Louis) and $25 \mathrm{mg} / \mathrm{kg}$ pentobarbital sodium solution (Abbott Laboratories, North Chicago, IL) given I.P. and were maintained with supplemental injections of a $100 \mathrm{mg} / \mathrm{kg}$ chloral hydrate $-10 \mathrm{mg} / \mathrm{kg}$ pentobarbital. Animals were initially positioned within a stereotaxic frame with the head placed at a $15^{\circ} \mathrm{C}$ angle (nose down). A small craniotomy was performed at $1.2 \mathrm{~mm}$ lateral and $3.6 \mathrm{~mm}$ caudal to the intersection of the midline and lambda. A 26-gauge hypodermic needle containing a tungsten electrode (UESMEESELNNE, FHC, Bowdoinham, $\mathrm{ME} ; 10 \mathrm{M} \Omega$ ), which extended $2.5 \mathrm{~mm}$ beyond the cannula, was advanced to locate the LC ( $\sim 6.0 \mathrm{~mm}$ ventrally). LC neurons were identified using previously described criteria (Devilbiss and Waterhouse, 2004). Once the lateral edge of the LC was located, the skull opening was sealed with Gelfoam, the cannula was attached to the skull with dental acrylic, and the electrode was removed from within the cannula. The head was then reoriented to a flat skull position and additional openings were made at $-3.3 \mathrm{~A} / \mathrm{P}$ and $-2.8 \mathrm{M} / \mathrm{L}$ and at $-2.5 \mathrm{~A} / \mathrm{P}$ and $-5.8 \mathrm{M} / \mathrm{L}$ (relative to bregma) for placement of VPm thalamus and BF cortex electrodes. Neuronal activity was monitored as electrodes were slowly lowered to evaluate neural responses to manual deflection of the C3 whisker and to confirm that the C3 whisker was the principal whisker for the majority of recorded neurons. A whisker-stimulating electrode was placed under the skin, terminating at the base of the C3 whisker follicle or the whisker activating the largest number of neurons of the BF cortex or VPm thalamus. When completed, the dura was covered with Gelfoam, each probe was fixed into position to the skull with dental cement, and the animal allowed to recover for 5-10 days.

\section{Infusions and Recording Sessions}

Animals were habituated to the experimenter, the experimental procedure (whisker pad stimulation), and the testing chamber environment for at least $2 \mathrm{~h}$ prior to experimentation. The testing environment was a Plexiglas chamber $\left(32 \times 32 \times 40 \mathrm{~cm}^{3}\right)$ contained within a sound-attenuated, ventilated outer chamber with masking white noise $(80 \mathrm{~dB})$. In the center of the top panel of the inner and outer chamber, a $10-\mathrm{cm}$ hole permitted infusion lines and electrophysiological cables to exit the chamber. A second hole in the front of the chamber permitted videotaping of the animal. Single units were discriminated from neural activity using a template-matching algorithm as previously described (Devilbiss and Waterhouse, 2004; Devilbiss et al, 2006). During habituation to the chamber, repeated uniform biphasic current pulses were delivered to whisker pad electrodes, adjusted to elicit stimulus-evoked responses from at least $50 \%$ of the recorded units and titrated for each animal to be threshold for eliciting movement from the C3 whisker.

Ovine CRF (Sigma-Aldrich, St Louis, MO) was dissolved in artificial cerebrospinal fluid (aCSF) at a concentration of $1.0 \mu \mathrm{g} / \mu \mathrm{l}$, aliquoted into $1.0 \mu \mathrm{l}$ samples, and stored at $-80^{\circ} \mathrm{C}$. On testing day, a 33-gauge infusion needle containing CRF was inserted into the 26-gauge cannula extending $2.5 \mathrm{~mm}$ beyond the depth of the cannula and secured via the plastic threading of the cannula. The needle was connected to a $10-\mu l$ syringe mounted on an infusion pump (Harvard Apparatus, South Natick, MA) located outside the recording chamber. Infusions of aCSF and CRF (30 or $300 \mathrm{nl}$ ) were used in these studies. For CRF, this resulted in a peri-LC dose of 30 or $300 \mathrm{ng}$. Infusion rates were 30 (30 $\mathrm{nl}$ infusions) and $100 \mathrm{nl} /$ $\min (300 \mathrm{nl}$ infusions) for both aCSF and CRF. Although no differences were observed between 30 and $100 \mathrm{nl}$ infusions of aCSF, all statistical comparisons for the effects of CRF were made against identical volumes of vehicle.

A recording session was comprised of three phases. First, baseline neuronal discharge activity was recorded for $45 \mathrm{~min}$ in 15-min blocks. Each block was comprised of a 10 -min interval of stimulation of the whisker pad $(0.5-3 \mathrm{~mA}$ at $0.5 \mathrm{~Hz}, 1 \mathrm{~ms}$ duration $\times 10 \mathrm{~min}=300$ stimulations) followed by 5 -min of recovery. Second, following baseline recordings, aCSF was administered by remote-control infusion and recordings continued in 15-min blocks for $1 \mathrm{~h}$ (four epochs). Third, following the vehicle recording, CRF was infused and recordings continued in 15-min blocks for a second hour (four epochs). Behavior was continually recorded throughout the entire experiment and stored on videotape with timestamps synchronized to the datacollection software. Only one recording session was made in a day, although rats received multiple recording sessions separated by at least three days (ie, different doses). The stability of recordings across weeks limited the possible number of recording sessions, thus not every animal received all doses of CRF. Only two animals participated in recording sessions with infusions of aCSF or CRF at anatomical control conditions.

\section{Analysis of Multi-Channel Electrophysiological Data}

The effects of CRF on VPm thalamic and BF cortical neuron discharge patterns were analyzed during periods of quiet 
resting as described in previous reports (Devilbiss and Waterhouse, 2004; Devilbiss et al, 2006). Videotape recordings were used to identify epochs of quiet resting, defined as a recumbent, head-raised position. Additionally, recorded waveforms were reexamined using pre-established criteria to verify that individual waveforms originated from a single neuron. These well-described off-line criteria included characterization of unit waveform properties and spike train discharge patterns (Figure 1d); (1) variability of peak voltage of the waveform, (2) variability of waveform slopes, (3) separability of scattergram clusters of the waveform's first two principal components (PC), and (4) refractory period analysis using the spike train auto-correlegram (Devilbiss and Waterhouse, 2004).

Peri-stimulus time histograms (PSTHs) were generated from spike train data acquired during quiet resting periods of baseline and post-infusion 15-min epochs. Analysis was restricted to this behavior to avoid arousal-related and behavior-related changes in LC and somatosensory neuronal discharge (Berridge and Foote, 1991; Devilbiss and Waterhouse, 2004; Aston-Jones and Bloom, 1981). The number of stimuli used to generate PSTHs for each 15-min epoch was set by determining the maximum possible number of stimuli occurring across all quiet resting periods of each 15-min epoch. From each PSTH, the spontaneous discharge rate was quantified as the mean discharge rate between 1 and $100 \mathrm{~ms}$ pre-stimulus. Whisker-pad stimulusevoked discharge was calculated as the mean probability of discharge calculated within a $30-\mathrm{ms}$ response window (4 to $34 \mathrm{~ms}$ post-stimulus) minus the mean spontaneous discharge probability. Additionally, the signal-to-noise ratio (SNR) was calculated as a $Z$-score $\left(\frac{\mathrm{x}-\mu}{\sigma}\right)$ such that $\mathrm{x}$ is the evoked-response, $\mu$ is the mean spontaneous discharge rate, and $\sigma$ is the standard deviation of the spontaneous discharge. Changes in spontaneous discharge, sensoryevoked discharge, or SNR during post-vehicle (aCSF) or post-CRF conditions were expressed as a percent of baseline for each neuron and then averaged across recordings. Data were analyzed using ANOVA and two-way repeatedmeasures ANOVA (time and treatment). Post-hoc comparisons were performed using the Fisher LSD test.

\section{Histology}

Placements of the recording microelectrodes within the thalamus and cortex and LC-infusion needle tract were verified histologically. Electrode-recording placements were visualized by a Prussian blue reaction following passing $60 \mu \mathrm{A}$ of cathodal current through each electrode for $45 \mathrm{~s}$. Animals were perfused with $0.9 \%$ saline followed by a $10 \%$ formalin solution containing 5\% potassium ferrocyanide. Coronal $80 \mu \mathrm{m}$ sections were cut on a freezing microtome, mounted and cover slipped following removal and storage for $72 \mathrm{~h}$ in phosphate buffer containing 15\% sucrose (see Figure 1).

\section{RESULTS}

In five animals, action-potential waveforms were recorded from the VPm thalamus and BF cortex in awake, unrestrained rats (Figure 1). Electrode locations within individual VPm barreloids and BF cortical barrels were mapped during surgery with mechanical displacement of a single whisker on the contralateral muzzle. Postmortem examination of coronal tissue sections revealed consistent placement of recording electrodes within the VPm thalamus or within layer $\mathrm{V}$ of the BF cortex. Neural activity from single units was discriminated, recorded, and analyzed from $121 \mathrm{VPm}$ thalamic and $78 \mathrm{BF}$ cortical cells (Figure 1d) with an average of 40 neurons/animal (range 11-45) across the VPm thalamus and BF cortex that were suitable for subsequent analysis. All the cells of this group were responsive to nonnoxious electrical stimulation of the contralateral whisker pad. Under awake, quiet resting control conditions, VPm neurons exhibited a mean spontaneous discharge rate of $2.12 \pm 0.93 \mathrm{~Hz}$ (SEM) and an average whisker-pad stimulusevoked discharge of $33.96 \pm 15.66 \mathrm{~Hz}$. Likewise under these behavioral conditions, $\mathrm{BF}$ cortical neurons demonstrated a mean spontaneous rate of $0.40 \pm 0.15 \mathrm{~Hz}$ and an average evoked-discharge rate of $20.62 \pm 12.12 \mathrm{~Hz}$.

\section{Dose-Dependent Effects of Peri-LC Infusions of CRF on Spontaneous and Sensory-Evoked Discharge of VPm Thalamic and BF Cortical Neurons}

To initially characterize the effects of peri-LC infusions of CRF on discharge patterns of VPm thalamus and BF cortex neurons, PSTHs were used to compare spontaneous and stimulus-evoked discharge of individual thalamic and cortical cells before and after infusions of vehicle and $300 \mathrm{ng}$ CRF. For the illustrative cases shown in Figure 2, vehicle (aCSF) infusions had little effect on evoked or spontaneous discharge. In contrast, CRF suppressed stimulus-evoked discharge of both VPm thalamic neurons (34\% and $59 \%$ of baseline, respectively, for Figure $2 \mathrm{a}$ and $\mathrm{b}$ ) and BF cortical neurons (33\% and $37 \%$ of baseline, respectively, for Figure $2 c$ and d). Peri-LC administration of CRF also increased the spontaneous discharge rate of these thalamic and cortical example cases. Spontaneous discharge increased by 457 and $600 \%$ for these two VPm thalamic neurons and 200 and $224 \%$ for these BF cortical neurons. Together, the reduction of stimulus-evoked discharge and the elevation of spontaneous firing rate resulted in a profound suppression of the SNR of these VPm thalamic and BF cortical neurons (89.3-92.5\% and 67.6-73.5\%, respectively). Recovery from these effects for these neurons began $30 \mathrm{~min}$ after peri-LC CRF infusions. This time course is consistent with previously described effects of CRF on LC neural activity (Curtis et al, 1997).

The effects of peri-LC infusions of CRF on signal transmission through this sensory pathway were further quantified by determining the dose-dependency of CRF actions for all recorded VPm thalamic and BF cortical neurons $15-30 \mathrm{~min}$ post infusion (Figure 3). Peri-LC CRF affected spontaneous activity of VPm thalamic neurons in a dose-dependent manner (ANOVA; Dose, $\mathrm{F}(1,39)=19.01$, $p<0.001)$. The highest dose of CRF tested significantly increased spontaneous discharge rates (332\% of baseline). However, neither the lower dose of CRF nor aCSF were effective at modulating spontaneous activity (Figure $3 \mathrm{a}$ ). The spontaneous discharge rate of $\mathrm{BF}$ cortical neurons was also dose-dependently increased by the highest CRF dose tested (ANOVA; Dose, $\mathrm{F}(1,33)=7.10, p=0.012$ ), with the highest dose of CRF increasing BF cortical spontaneous 


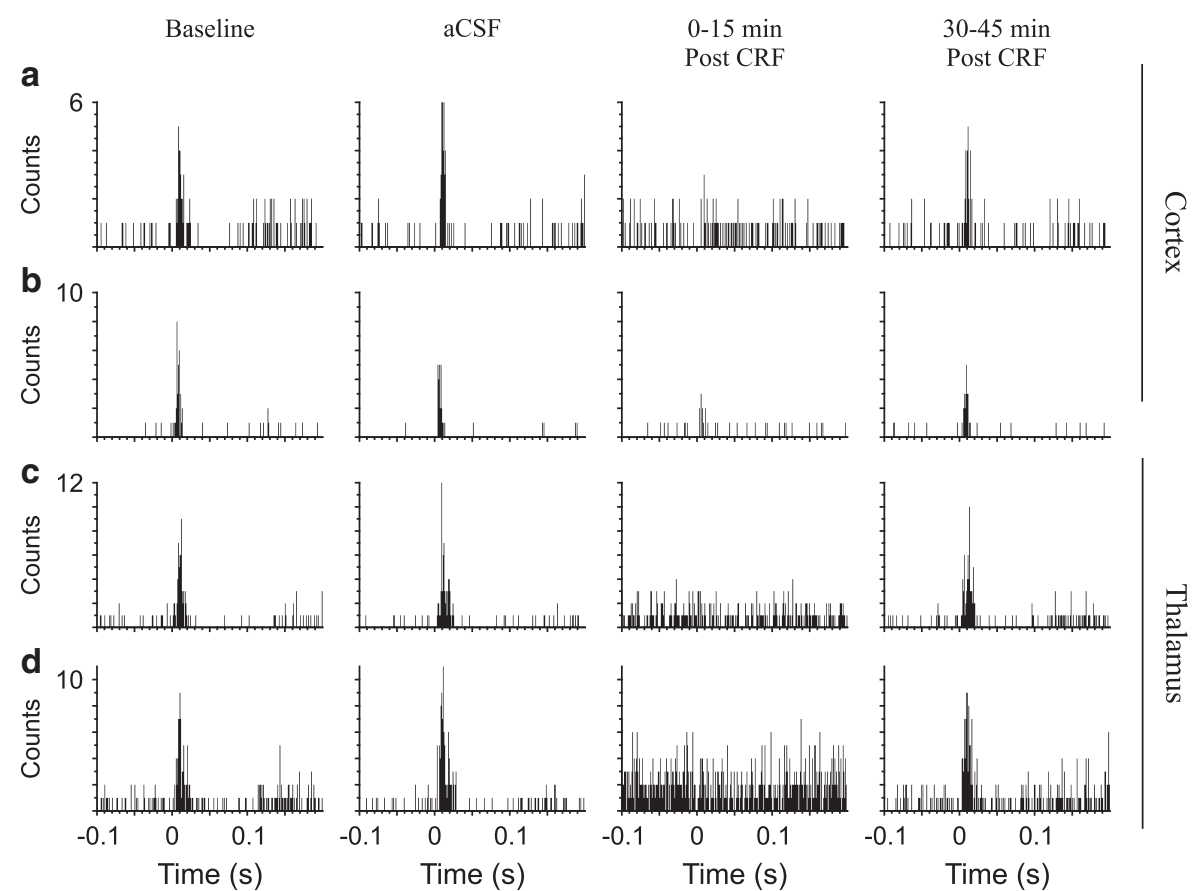

Figure 2 Peri-stimulus time histograms (PSTHs) illustrate the effects of peri-LC infusions of CRF on VPm thalamic and BF cortical neuronal activity. Neuronal discharge patterns of representative individual neurons are plotted for two cortical neurons (a and b) and two thalamic neurons (c and d) for a 15-min baseline period, 0-15 min following artificial cerebrospinal fluid (aCSF) infusion, as well as 0-15 and 30-45 min post CRF infusion. CRF (300 ng) decreased evoked-discharge of VPm thalamic and BF cortical neurons but increased spontaneous firing rates. VPm thalamic neurons exhibited larger increases in spontaneous activity than that observed in the BF cortex. The abscissae depict time before and after the presentation of whisker pad stimuli (occurring at $0 \mathrm{~ms}$ ). The ordinates represent the spike counts per time bin (I ms).

firing rates $181 \%$ of baseline. Further analysis indicated that the high dose of CRF produced a significantly greater increase in spontaneous discharge for VPm thalamic vs BF cortical neurons (Figure 3a).

In contrast to that seen for spontaneous discharge, periLC infusions of CRF suppressed stimulus-evoked discharge (Figure $3 \mathrm{~b}$ ). For VPm thalamic neurons, this suppression was statistically significant (rmANOVA; Treat ${ }^{*}$ Dose, $\mathrm{F}(4,204)=3.98, p=0.004)$ at the highest dose of CRF (69\% reduction from baseline conditions). Stimulus-evoked discharge of BF cortical neurons was also significantly suppressed following the high dose of CRF (59\% of baseline; rmANOVA; Treat ${ }^{\star}$ Dose, $\left.F(4,152)=2.66, p=0.034\right)$. Neither aCSF nor the low dose of CRF (30 ng) altered somatosensory-evoked responses of VPm thalamic or BF cortical neurons. A comparison of effects between the thalamus and cortex revealed that the high dose of CRF produced a greater suppression of stimulus-evoked discharge in the VPm thalamus. However, this difference was not statistically significant.

Measures of the SNR of neuronal responses along this somatosensory pathway were also suppressed by peri-LC CRF. The SNR of VPm thalamic neurons was significantly suppressed at the high dose of CRF (rmANOVA; Treat ${ }^{\star}$ Dose, $\mathrm{F}(2,88)=6.16, p=0.003$ ) (Figure $3 \mathrm{c}$ ). Likewise, the high dose of CRF suppressed the SNR of BF cortical neurons from baseline conditions ( $t$-test; $p=0.0037)$, but was insufficient to generate a statistical difference in the omnibus rmANOVA (Treat ${ }^{\star}$ Dose, $\mathrm{F}(2,36)=1.35, p=0.27$ ). A comparison of these regions indicated that the highest dose of CRF produced a significantly greater reduction in the SNR for VPm thalamic neurons than that observed for BF cortical neurons.

\section{Time-Course of CRF Effects on Spontaneous and Stimulus-Evoked Discharge of VPm Thalamic and BF Cortical Neurons}

To characterize the time-course of CRF actions, the effects of peri-LC infusions of $300 \mathrm{ng}$ CRF or aCSF on spontaneous activity, stimulus-evoked discharge, and the SNR of whisker-pad stimulus-evoked responses of VPm thalamic and $\mathrm{BF}$ cortical neurons were quantified in $15 \mathrm{~min}$ intervals following infusions. CRF-induced increases in spontaneous discharge rates of VPm thalamic neurons reached a maximum between 15-30 min post-infusion (Figure 4a). Repeated-measures ANOVA indicated a dose by time interaction (Treat ${ }^{\star}$ Dose, $\mathrm{F}(4,172)=5.97, p<0.001$ ). Posthoc analysis confirmed that spontaneous discharge for VPm thalamic neurons, elevated by CRF between 0 and $45 \mathrm{~min}$, were both different from baseline and aCSF infusions (Peak $2.82 \pm 0.41 \mathrm{~Hz}(\mathrm{SEM})$ ). Spontaneous discharge rates of BF cortical neurons were also elevated, but no dose-time interaction was present (Treat ${ }^{\star}$ Dose, $\mathrm{F}(4,176)=1.81, p=$ $0.128)$ (Peak $=0.49 \pm 0.08 \mathrm{~Hz})$. However, the main effect of dose in this analysis was significant (Dose, $\mathrm{F}(1,44)=8.06$, $p=0.007)$ and a significant increase in spontaneous activity of $\mathrm{BF}$ cortical neurons was observed for the first $30 \mathrm{~min}$ post-CRF infusion relative to baseline conditions. Finally, a significant difference was observed between CRF effects on the spontaneous activity of VPm thalamic neurons 

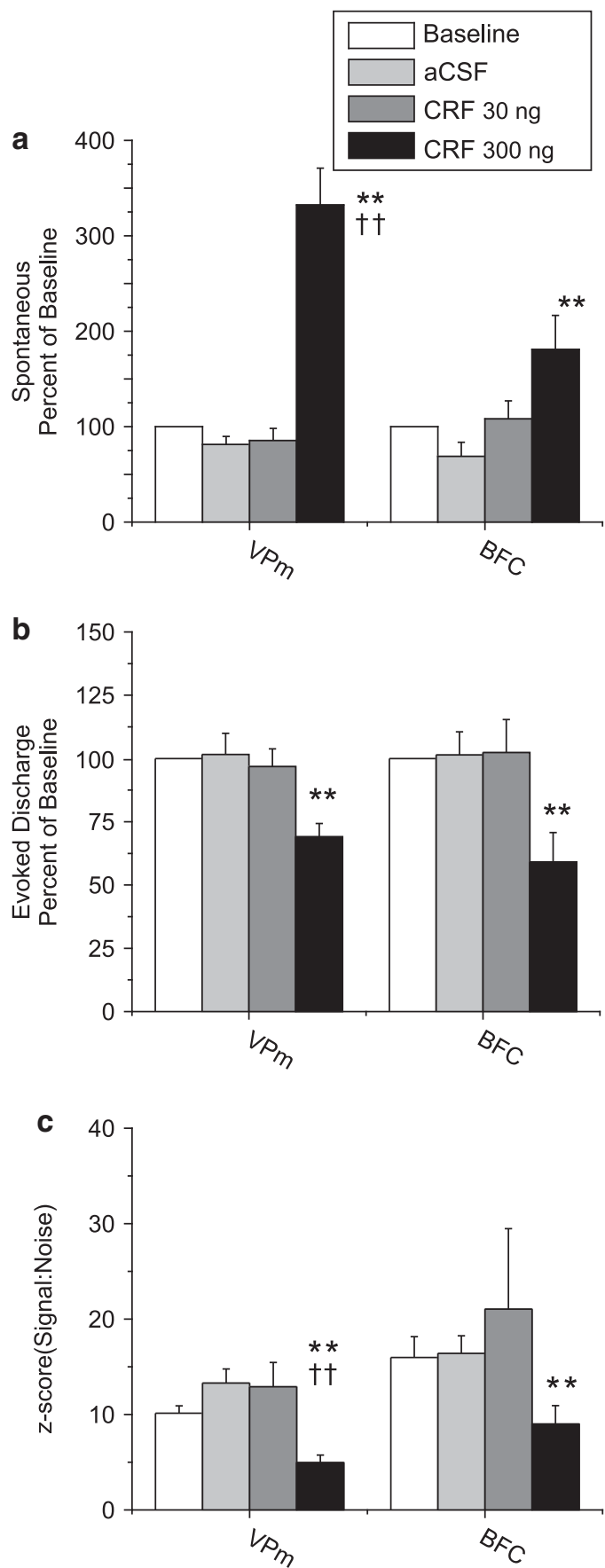

Figure 3 Effects of peri-LC infusions of CRF on spontaneous and stimulus-evoked discharge of VPm thalamic or BF cortical neurons. (a) Spontaneous activity of VPm thalamic neurons is dose-dependently increased 15-30 min following 30 or $300 \mathrm{ng}$ peri-LC CRF infusions. Additionally, neurons of the VPm thalamus exhibit a greater increase in spontaneous activity following CRF administration compared with the BF cortex. (b) Whisker-stimulus evoked discharge of VPm thalamus and BF cortex neurons is suppressed with the highest dose of CRF. A similar degree of suppression is seen in the thalamus and cortex. (c) SNR of sensory-evoked discharge in VPm thalamus and BF cortex is reduced by peri-LC CRF infusions in a dose-dependent manner. The Z-score of the mean evoked-response compared with background was used to calculate the SNR. The VPm thalamus demonstrated a greater suppression of SNR following CRF administration than was observed for the BF cortex. $(n=5$ animals, neurons $=88 / 55 \mathrm{VPm} / \mathrm{BFC}$; LSD comparison with baseline: ** $p<0.0$ I; LSD comparison with BFC $p<0.01$; all data presented as mean \pm SEM). compared with $\mathrm{BF}$ cortical neurons at $15-30$ min post periLC CRF infusion.

A similar time course of effects was observed for stimulusevoked responses. Evoked responses of VPm thalamic neurons were maximally suppressed by peri-LC infusions of CRF between 15 and $30 \mathrm{~min}$ post infusion (Figure $4 \mathrm{~b}$ ). This suppression was statistically significant for the first $30 \mathrm{~min}$ following peri-LC infusions of the highest dose of CRF $\left(\right.$ rmANOVA; Treat ${ }^{*}$ Dose, $\left.F(4,276)=2.68, p=0.032\right)$. Similarly, BF cortical neurons exhibited a significant suppression of whisker-pad stimulus-evoked responses $15-30 \mathrm{~min}$ post infusion (ANOVA; Dose, $\mathrm{F}(1,52)=4.62, p=0.036$ ).

CRF suppression of the SNR of somatosensory-evoked responses also occurred over a similar time-course (Figure 4c). Repeated-measures ANOVA indicated a significant dose by time interaction for both VPm thalamic neurons $(\mathrm{F}(4,164)=3.80, \quad p=0.0055)$ and $\mathrm{BF}$ cortical neurons $(\mathrm{F}(4,92)=2.80, p=0.030)$. Further analysis indicated a significant suppression of the SNR by CRF at the 30min time point, which returned to baseline levels within $1 \mathrm{~h}$. Comparisons between the suppressant effects of CRF on SNR for VPm thalamic and BF cortical neurons indicated no regional interaction effect. As such, although VPm thalamic neurons demonstrated an overall lower SNR following CRF administration, the degree to which CRF suppressed VPm thalamic neuron responses was similar to its suppression of BF cortical SNR for sensory-evoked discharges.

\section{Anatomical Specificity of CRF Effects on Spontaneous and Stimulus-Evoked Discharge of VPm Thalamic and BF Cortical Neurons}

Additional studies tested whether the neuromodulatory effects of CRF were limited to infusions proximal to the LC. CRF infusions were made $900-1000 \mu \mathrm{m}$ lateral to the LC $(750-850 \mu \mathrm{m}$ lateral to peri-LC infusion sites). Lateral infusions of either 150 or $300 \mathrm{ng}$ CRF did not alter spontaneous discharge rates or responses to somatosensory input for either VPm thalamic or BF cortical neurons (Figure 5). Repeated-measures ANOVA indicated no significant effects of lateral infusions of the highest dose of CRF relative to peri-LC infusions of aCSF for either spontaneous discharge rates of VPm thalamic neurons (Time Treat, $\mathrm{F}(3,192)=0.48, p>0.05)$, evoked-responses (Time* Treat, $\mathrm{F}(3,156)=0.14, p>0.05)$, or SNR calculations $\left(\right.$ Time $^{\star}$ Treat, $\left.F(3,153)=2.44, p>0.05\right)$. Similarly, no significant difference was observed between the discharge activity of BF cortical neurons following lateral CRF infusions compared with peri-LC infusions of aCSF (Time*Treat, rmANOVA(spontaneous), $\mathrm{F}(3,150)=0.82$, $p>0.05$; rmANOVA(evoked), $\mathrm{F}(3,105)=0.56, p>0.05$; and rmANOVA(SNR), $\mathrm{F}(3,84)=0.66, p>0.05)$. BF cortex SNR was slightly lower before and after lateral LC infusions of CRF, however, no change in SNR was observed following lateral CRF infusion.

\section{DISCUSSION}

The current study found that CRF acts at the LC to modulate spontaneous discharge rates and sensory-stimulus response properties within forebrain sensory circuits of 

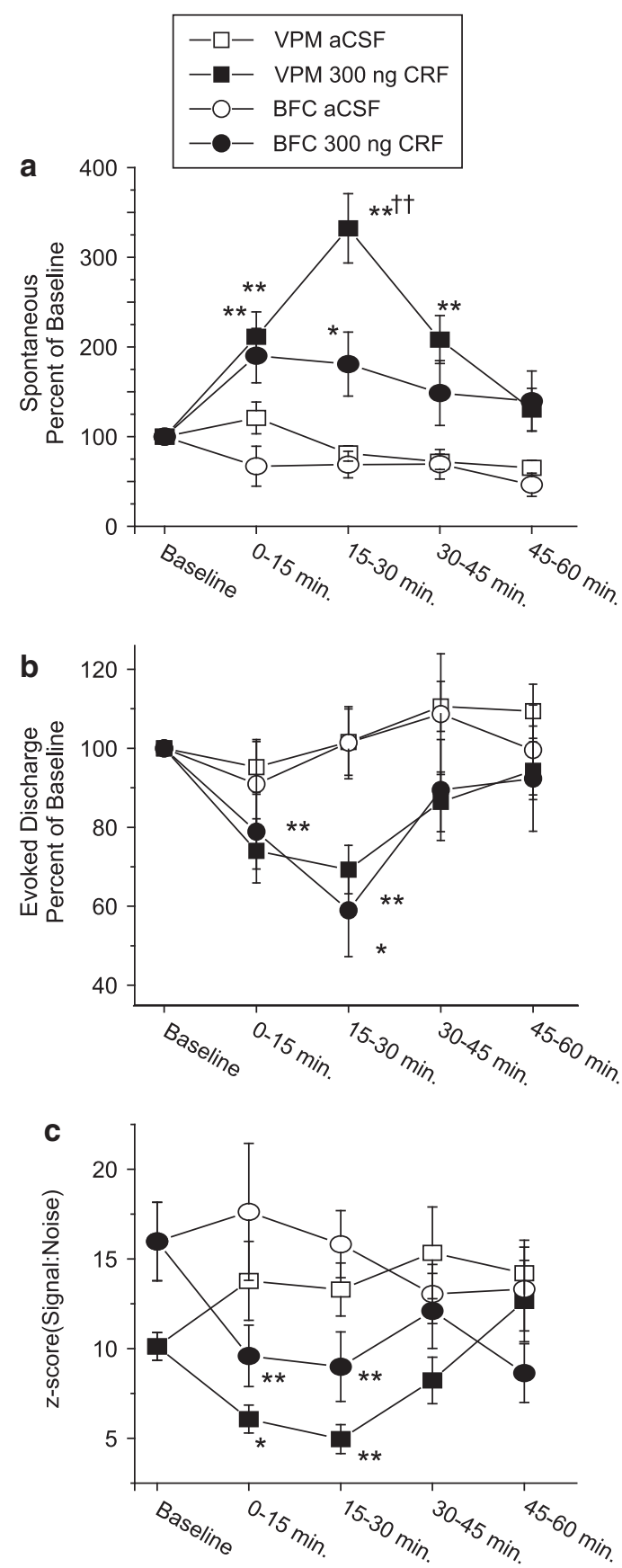

Figure 4 Time course of CRF effects for VPm thalamic or BF cortical neurons discharge patterns. The modulatory actions of vehicle (aCSF) and the highest dose of CRF ( $300 \mathrm{ng}$ ) were plotted in 15 min intervals for I h following peri-LC infusion. (a) The neuromodulatory effects of peri-LC CRF infusions on spontaneous activity peaked within $15 \mathrm{~min}$ in the BF cortex, whereas VPm thalamic neurons exhibited the largest increase in spontaneous activity at 15-30 post-infusion. (b) Modulation of whisker-stimulus evoked discharge by peri-LC CRF infusions occurred over a similar time course as changes in spontaneous activity. Maximum suppression of evoked discharge within VPm thalamus and BF cortex occurred between I5-30 post-infusion. (c) SNR calculations indicated that the peak effect occurred 15-30 min following CRF infusion. ( $n=5$ animals, neurons $=88 / 55 \mathrm{VPm} / \mathrm{BFC}$; LSD comparison with baseline: ${ }^{*} p<0.05$, ** $p<0.0$ I; LSD comparison with BFC ${ }^{\dagger \dagger} p<0.0$ I; all data presented as mean \pm SEM)

awake, freely moving animals. Specifically, peri-LC infusions of CRF dose-dependently increased spontaneous firing rates of individual VPm thalamic and BF cortical somatosensory neurons while simultaneously suppressing sensory stimulus-evoked discharge. The profound reduction in the SNR of stimulus-evoked responses suggests that forebrain somatosensory signal processing is altered during conditions where CRF is released at the LC, including stress. Such an action could serve an important adaptive response to an immediate, unexpected stressor. Furthermore, these observations suggest that disruptions in information processing within the sensory thalamus and cortex may contribute to stress-related deficiencies in sensorimotor gating. Persistence of such effects could contribute to abnormalities of sensory information processing seen in stress-related psychiatric disorders.

\section{Technical Considerations}

Peri-LC infusions were utilized in the current studies to minimize damage to LC neurons that project to the VPm thalamus and BF cortex (Simpson et al, 1997) and to target CRF receptors located on dendrites of LC cells (Van Bockstaele et al, 1996). Recordings in the awake, freely moving animal precluded the use of glass micropipettes positioned directly within the LC (Curtis et al, 1997), and placement of a 33-gauge needle into the LC likely results in a substantial damage to this nucleus (see Berridge and Foote, 1991). Pharmacological mapping studies within the pons demonstrate that selective manipulation of LC neuronal discharge activity is achieved with the approach utilized in the current study, with an effective radii regularly in the range of $\sim 400-500 \mu \mathrm{m}$ when small infusion volumes are used (Berridge and Foote, 1991; Page et al, 1993; Zhang et al, 1990). Consistent with this, we found that CRF infusions $900 \mu \mathrm{m}$ distal to the LC were ineffective in producing modulation of discharge activity within the somatosensory system.

In the current study, $300 \mathrm{ng}$ of CRF elicited a significant change in neuronal activity of the VPm thalamus and BF cortex. We estimated CRF concentrations at the LC following diffusion from the peri-LC infusion site using the upper bounds estimate (Newton's inverse square law) and with more realistic models that account for the diffusion coefficient of CRF, volume fraction, and tortuosity (Nicholson, 2001; Shorten and Wall, 2001). These analyses indicate that our peri-LC infusions resulted in CRF concentrations within the LC nucleus nearly identical to previous studies that directly infused CRF within the LC (Curtis et al, 1997; Snyder et al, 2012). Specifically, following $300 \mathrm{ng}$ CRF infusions, maximal estimates of CRF were 30 and $3 \mathrm{ng}$ at distances of 32 and $110 \mu \mathrm{m}$ from the infusion needle, respectively, using the inverse square method. Using the Crank-Nicholson method, peak concentrations of $3 \mathrm{ng}$ CRF doses were calculated for a distance of $32 \mu \mathrm{m}$ from the infusion needle tip (Nicholson, 2001; Shorten and Wall, 2001). Together, the above observations provide strong evidence that the neuromodulatory effects of CRF infusions resulted from action at the LC and not from actions at other pontine nuclei.

Arousal-related changes in LC-NE modulatory tone of the somatosensory system were controlled in these studies by limiting analyses to quietly-resting behavioral states. Baseline LC discharge rates are highly correlated with the level of arousal (Aston-Jones and Bloom, 1981). Likewise, responses 

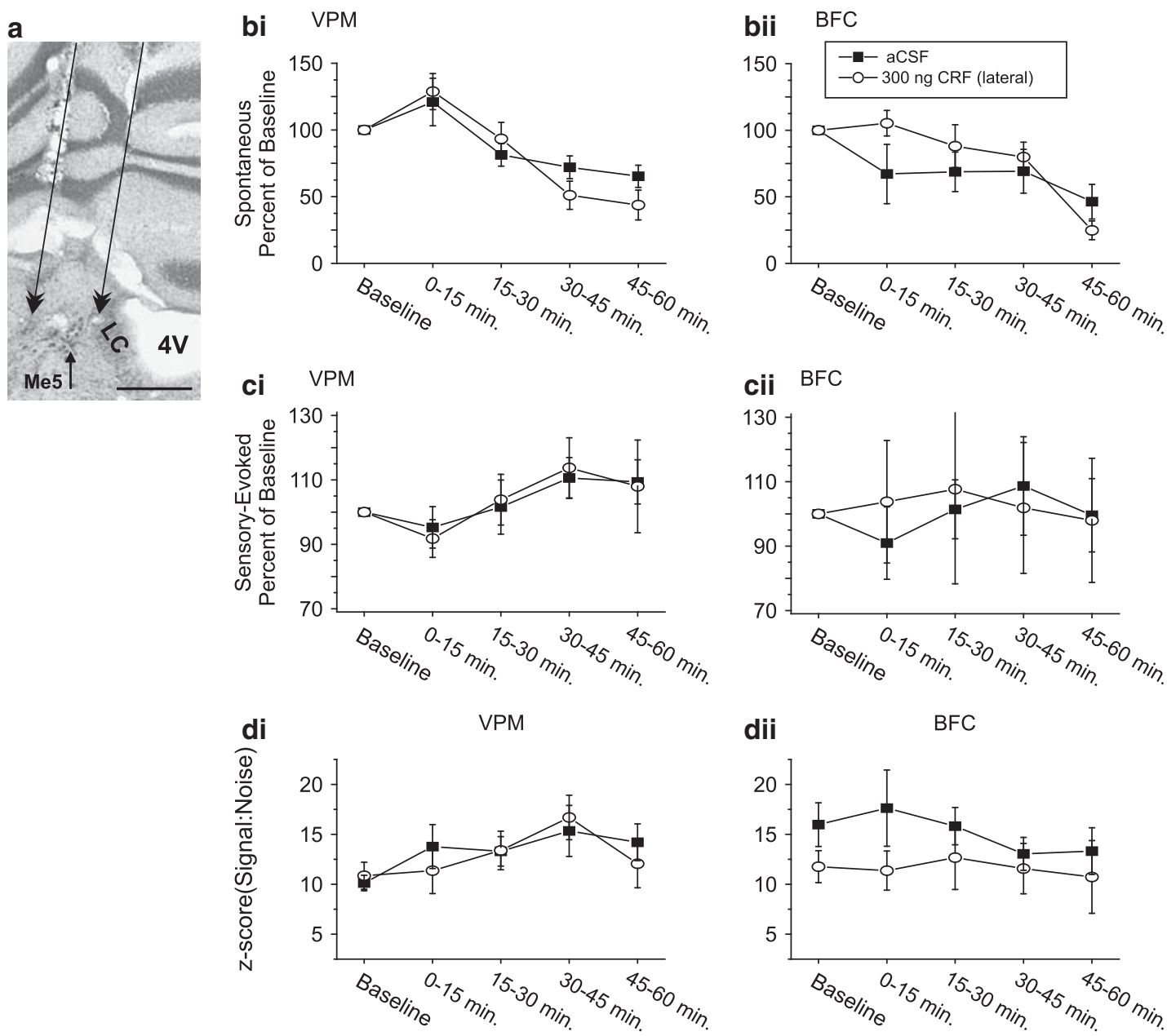

Figure 5 Determination of CRF site of action. The effects of peri-LC infusions of CRF were compared with infusions lateral to the LC. (a) $\times 40$ photomicrograph illustrates two needle tracts generated from a peri-LC and lateral infusion site. Bar $=1 \mathrm{~mm}$. (b) Spontaneous discharge calculated as a percent of baseline for VPm (bi) and BF cortical (bii) neurons after peri-LC infusions of vehicle, CRF (300 ng), or lateral LC infusions of CRF (300 ng). (c) Lateral infusions of CRF distal to the LC did not effect whisker stimulus-evoked discharge. (d) SNR calculations also demonstrated no change from baseline for lateral infusions of CRF. Although the SNR was slightly lower for BF cortical neurons recorded before and after lateral LC infusions, lateral LC CRF infusions did not significantly alter the responses of these neurons to sensory stimuli. ( $n=2$ animals, neurons $=33 / 23 \mathrm{VPm} / \mathrm{BFC}$; LSD comparison with baseline; all data presented as mean \pm SEM).

to environmental objects by the cortical and thalamic somatosensory pathway neurons are also dependent on arousal levels (Fanselow and Nicolelis, 1999). During repose, the LC is not tonically activated by endogenous CRF (Page and Abercrombie, 1999). Nonetheless, CRF has been shown to be more potent and have a longer duration of action in increasing LC firing rates of unanesthetized $v s$ anesthetized rats (Valentino and Foote, 1988). As the effects of peri-LC infusions of CRF on neuronal activity of the VPm thalamus and BF cortex may differ between waking behavioral states, this will be examined in future studies.

\section{Neuromodulation of Forebrain Circuitry by CRF Actions at the LC}

Considerable evidence suggests that during stress, CRF acts directly at the level of the LC to increase neuronal discharge rates of LC-NE neurons (Valentino and Van Bockstaele, 2008) and coordinate central neurophysiological responses in stress (Bale and Vale, 2004; Hauger et al, 2009; Owens and Nemeroff, 1991). CRF is released at the LC during stress and it is estimated that levels of CRF in the LC may be increased two-fold during exposure to stressors (Chappell et al, 1986). Moreover, activation of the LC projection system by CRF can elicit a two-fold increase in spontaneous LC discharge rates (Curtis et al, 1997; Page and Abercrombie, 1999). The broad distribution of LC fibers throughout the CNS is well known and activation of this system by CRF can evoke an approximate 1.6-fold increase in NE release throughout the neuraxis (Page and Abercrombie, 1999; Smagin et al, 1995). Thus, the CRF-LC-NE circuit is a major stress-response pathway that is well positioned to regulate forebrain functions in stress.

In the current study, CRF suppressed sensory-evoked discharge of VPm thalamic and BF cortical neurons. In comparison, prior studies have shown that increasing levels of LC-NE output can facilitate sensory-evoked responses of VPm thalamic and BF cortical neurons according to an inverted-U dose-response relationship (Devilbiss and Waterhouse, 2004; Devilbiss et al, 2006). At the highest levels of LC 
output $(5 \mathrm{~Hz})$, associated with a 1.6-fold increase in $\mathrm{NE}$ release within the VPm thalamus, sensory-evoked discharge is suppressed from optimal responsiveness to near minimal or moderate responsiveness as observed under baseline conditions (Devilbiss and Waterhouse, 2004). Together these observations suggest that during stress and conditions of elevated CRF neurotransmission, the neuromodulatory tone within LC targets is in the upper range of the inverted- $U$ shaped curve.

Both peri-LC infusions of CRF and high levels of tonic LC$\mathrm{NE}$ output can suppress evoked-activity of thalamic and cortical target neurons. However, peri-LC infusions of CRF also produced robust increases in the spontaneous firing rate of VPm thalamic and BF cortical neurons. These effects resulted in a strong inhibition in SNR measures. However, together these actions generally do not occur simultaneously with iontophoretic application of NE on target somatosensory neurons or direct stimulation of the LC-NE system (Armstrong-James and Fox, 1983; Devilbiss and Waterhouse, 2004; Devilbiss et al, 2006). It is possible that release of colocalized neuroactive peptides, including galanin and neuropeptide Y, during CRF activation of the LC could partially explain these differences (Bijak, 2000; Pieribone et al, 1998; Schlifke et al, 2006; Simpson et al, 1999; Verhage et al, 1991). However, prior evidence indicates that galanin and neuropeptide Y generally inhibit spontaneous target neuron discharge activity, suggesting that these neuropeptides may not participate in the forebrain modulatory effects of CRF activation of the LC (Bijak, 2000; Pieribone et al, 1998; Schlifke et al, 2006). Regardless of the underlying mechanism, the current observations demonstrate that CRF-dependent activation of the LC exerts unique modulatory actions in forebrain circuits relative to direct application of $\mathrm{NE}$ or electrical activation of the LC in a range associated with active waking.

Finally, we found that the modulatory effects of CRF observed within the cortex were not simply an amplification of effects occurring in the thalamus. Instead, the large modulatory effects observed within the VPm thalamus were constrained and minimized within the BF cortex. This finding supports the hypothesis that although LC output can simultaneously have an impact on thalamic and cortical activity, the LC-NE has unique consequences on signal processing operations at each level of an ascending sensory network (Devilbiss and Waterhouse, 2004; Devilbiss et al, 2006). LC neurons innervate the entire rat-ascending trigeminal pathway (Simpson et al, 1997; Swanson and Hartman, 1975) and can simultaneously modulate thalamic and cortical neuronal discharge. However, differences in ipsilateral LC projection densities between the VPm thalamus and BF cortex (Simpson et al, 1997) may, in part, explain these quantitative differences in peri-LC CRF modulation of sensory-evoked discharge of VPm thalamic and $\mathrm{BF}$ cortical neurons.

\section{Functional Relevance}

Suppression of neuronal responsiveness to excitatory synaptic input may reflect an organism-wide strategy to limit certain forms of sensory signal transmission during stressful situations (ie, those that elicit the highest levels of tonic LC discharge; Devilbiss and Waterhouse, 2004;
Devilbiss et al, 2006). This is supported by the current findings that CRF, acting at the LC, suppresses sensoryevoked excitatory neurotransmission and SNR measures within the somatosensory thalamus and cortex. Moreover, studies examining evoked-response potentials of electroenceplographic recordings provide further support for deficits in sensory signal processing during stress (Clark et al, 1986; Ermutlu et al, 2005; Miyazato et al, 2000).

Encoding and filtering sensory information are regulated, in part, by the LC-NE system (Hurley et al, 2004). Previous evidence suggests that suppression of sensory-evoked discharge alters sensory and perceptual processes by reducing the detectability of low-intensity punctate stimuli, similar to the current study (Rutter et al, 2005). Furthermore, prior studies have shown that shifts in filtering properties of the somatosensory circuitry, which suppress low-frequency signals while preserving high-frequency information (necessary for texture discrimination; Moore, 2004), result in a similar modulation of somatosensoryevoked discharge properties to those in the current study. Thus together, the combined suppression of sensory-evoked responses and elevated spontaneous discharge during CRF actions at the LC may serve a prominent role in sensory circuit function outside simple reductions in SNR measures. Under certain conditions, increased spontaneous firing is beneficial for sensory encoding. For example, when such spontaneous firing is correlated across neuronal ensembles, postsynaptic neurons receiving convergent inputs from these ensembles can take advantage of redundancies in this correlated noise (Schneidman et al, 2003) to enhance the separation of the encoded sensory signal from other neural activity. However, this and other noise-based mechanisms of signal processing are likely most relevant to encoding high-frequency sensory information. Future studies with more complex sensory stimuli will be required to address explicitly the impact of CRF and stress on information processing within this and other sensory systems. Nonetheless, the current studies demonstrate that CRF actions at the LC reduce the neuronal detectability of low-intensity, punctate sensory stimuli.

Considerable evidence from human and animal studies shows that stressors impair PPI, the ability of a weak sensory stimulus (prepulse) to inhibit (gate) a startle motor response to an intense stimulus (Braff et al, 2001; Grillon and Davis, 1997; Orr et al, 2002). Although the neural circuitry underlying PPI is well documented (Gresack and Risbrough, 2010; Swerdlow et al, 2001), the evidence implicating brain regions involved in the regulation of PPI continues to accumulate. Animal studies suggest that stressrelated deficits in PPI involve both the CRF and LC-NE systems (Alsene and Bakshi, 2011; Conti et al, 2002; Gresack and Risbrough, 2010). In these studies, central infusions of both $\mathrm{CRF}$ and $\mathrm{NE}$, as well as direct pharmacological activation of the LC, produce deficits in PPI. Together, these observations suggest that CRF actions at the LC represent an important modulatory pathway for stressrelated alterations in sensory signal processing.

The effects of stress, CRF, and LC-NE activation on sensory signal processing and modulation of PPI may be mediated by changes in neural function in any number of LC terminal fields. In the current study, we have used the wellcharacterized ascending trigeminal pathway to characterize 
the effects of peri-LC infusions of CRF on sensory-stimulus evoked response properties of individual neurons (Van der Loos, 1976; Simons, 1978; Waite, 1973; Simpson et al, 1997; Devilbiss and Waterhouse, 2004; Devilbiss et al, 2006). Notably, direct infusion of CRF into the BF cortex produced no change in the spontaneous or evoked discharge of these cells (Cahusac et al, 1998), suggesting that CRF may not have direct modulatory actions within the sensory cortex when released during stress. Together, the current results demonstrate that CRF actions at the LC fundamentally alter the manner in which sensory information is transmitted by the thalamic and cortical neurons. Somatosensory stimuli, as well as auditory stimuli, and a number of other sensory modalities can be used to elicit PPI (Braff et al, 2001). Similarities between modulation of response properties of neurons in the somatosensory pathway and auditory systems (Hurley et al, 2004) suggest that the current observations are directly translatable between these sensory modalities. Moreover, the well described projections from the somatosensory and auditory cortex to the prefrontal cortex and limbic structures suggest a circuit mechanism by which changes in sensory signal processing by the thalamus and sensory cortex could modulate PPI through 'top-down' mechanisms (Swerdlow et al, 2001).

\section{Clinical Implications}

Acute stress accounts for a majority of all work-place accidents and millions of disabling injuries a year (BarriosChoplin et al, 1998). Stress suppresses sensory stimulus detection and discrimination (Clark et al, 1986) and impairs cognitive abilities including sustained attention to sensory information. There is a substantial evidence for impairment in sensory processing in humans with stress-related psychiatric diseases, particularly PTSD (Orr et al, 2002). The present findings suggest that CRF actions at the LC may contribute to altered sensory information processing in stress and stress-related disorders.

\section{ACKNOWLEDGEMENTS}

This work was supported by the National Institutes of Health (MH069072 to DMD, NIH NS32461 to BDW, MH62359 to CWB, and MH40008 and DA09082 to RJV).

\section{DISCLOSURE}

BDW and RV declared no conflict of interest. DM Devilbiss is the founder of NexStep Biomarkers, LLC. NexStep Biomarkers had no role in study design, data collection and analysis, decision to publish, or preparation of the manuscript. NexStep Biomarkers does not employ anyone who worked on this project, hold patents related to this project, sell products related to this project, or provided consultation on this project. This manuscript provides no financial gain for NexStep Biomarkers. CW Berridge has received expert witness fees from Teva Pharmaceutical, Activis, Aurobindo Pharmaceuticals, Mylan Pharmaceuticals, and Apotex.

\section{REFERENCES}

Alsene KM, Bakshi VP (2011). Pharmacological stimulation of locus coeruleus reveals a new antipsychotic-responsive pathway for deficient sensorimotor gating. Neuropsychopharmacology 36: 1656-1667.

Armstrong-James M, Fox K (1983). Effects of ionophoresed noradrenaline on the spontaneous activity of neurones in rat primary somatosensory cortex. J Physiol 335: 427-447.

Aston-Jones G, Bloom FE (1981). Activity of norepinephrinecontaining locus coeruleus neurons in behaving rats anticipates fluctuations in the sleep-waking cycle. J Neurosci 1: 876-886.

Bale TL, Vale WW (2004). CRF and CRF receptors: role in stress responsivity and other behaviors. Annu Rev Pharmacol Toxicol 44: 525-557.

Barrios-Choplin B, McCraty R, Cryer B (1998). An inner quality approach to reducing stress and improving physical and emotional wellbeing at work. Stress Medicine 13: 193-201.

Berridge CW, Foote SL (1991). Effects of locus coeruleus activation on electroencephalographic activity in neocortex and hippocampus. J Neurosci 11: 3135-3145.

Bijak M (2000). Neuropeptide Y reduces epileptiform discharges and excitatory synaptic transmission in rat frontal cortex in vitro. Neuroscience 96: 487-494.

Braff DL, Geyer MA, Swerdlow NR (2001). Human studies of prepulse inhibition of startle: normal subjects, patient groups, and pharmacological studies. Psychopharmacology (Berl) 156: 234-258.

Cahusac PM, Castro MG, Robertson L, Lowenstein PR (1998). Electrophysiological evidence against a neurotransmitter role of corticotropin-releasing hormone $(\mathrm{CRH})$ in primary somatosensory cortex. Brain Res 793: 73-78.

Chappell PB, Smith MA, Kilts CD, Bissette G, Ritchie J, Anderson C et al (1986). Alterations in corticotropin-releasing factor-like immunoreactivity in discrete rat brain regions after acute and chronic stress. J Neurosci 6: 2908-2914.

Clark WC, Yang JC, Janal MN (1986). Altered pain and visual sensitivity in humans: the effects of acute and chronic stress. Ann N Y Acad Sci 467: 116-129.

Conti LH, Murry JD, Ruiz MA, Printz MP (2002). Effects of corticotropin-releasing factor on prepulse inhibition of the acoustic startle response in two rat strains. Psychopharmacology (Berl) 161: 296-303.

Curtis AL, Lechner SM, Pavcovich LA, Valentino RJ (1997). Activation of the locus coeruleus noradrenergic system by intracoerulear microinfusion of corticotropin-releasing factor: effects on discharge rate, cortical norepinephrine levels and cortical electroencephalographic activity. J Pharmacol Exp Ther 281: 163-172.

Devilbiss DM, Page ME, Waterhouse BD (2006). Locus ceruleus regulates sensory encoding by neurons and networks in waking animals. J Neurosci 26: 9860-9872.

Devilbiss DM, Waterhouse BD (2004). The effects of tonic locus ceruleus output on sensory-evoked responses of ventral posterior medial thalamic and barrel field cortical neurons in the awake rat. J Neurosci 24: 10773-10785.

Ermutlu MN, Karamursel S, Ugur EH, Senturk L, Gokhan N (2005). Effects of cold stress on early and late stimulus gating. Psychiatry Res 136: 201-209.

Fanselow EE, Nicolelis MA (1999). Behavioral modulation of tactile responses in the rat somatosensory system. J Neurosci 19: 7603-7616.

Gresack JE, Risbrough VB (2010). Corticotropin-releasing factor and noradrenergic signalling exert reciprocal control over startle reactivity. Int J Neuropsychopharmacol 14: 1-16.

Grillon C, Davis M (1997). Effects of stress and shock anticipation on prepulse inhibition of the startle reflex. Psychophysiology 34: 511-517. 
Hauger RL, Risbrough V, Oakley RH, Olivares-Reyes JA, Dautzenberg FM (2009). Role of CRF receptor signaling in stress vulnerability, anxiety, and depression. Ann N Y Acad Sci 1179: $120-143$.

Holstein DH, Vollenweider FX, Jancke L, Schopper C, Csomor PA (2010). P50 suppression, prepulse inhibition, and startle reactivity in the same patient cohort suffering from posttraumatic stress disorder. J Affect Disord 126: 188-197.

Hurley LM, Devilbiss DM, Waterhouse BD (2004). A matter of focus: monoaminergic modulation of stimulus coding in mammalian sensory networks. Curr Opin Neurobiol 14: 488-495.

Kawahara H, Kawahara Y, Westerink BH (2000). The role of afferents to the locus coeruleus in the handling stress-induced increase in the release of noradrenaline in the medial prefrontal cortex: a dual-probe microdialysis study in the rat brain. Eur $J$ Pharmacol 387: 279-286.

Miyazato H, Skinner RD, Garcia-Rill E (2000). Locus coeruleus involvement in the effects of immobilization stress on the p13 midlatency auditory evoked potential in the rat. Prog Neuropsychopharmacol Biol Psychiatry 24: 1177-1201.

Moore CI (2004). Frequency-dependent processing in the vibrissa sensory system. J Neurophysiol 91: 2390-2399.

Nicholson C (2001). Diffusion and related transport mechanisms in brain tissue. Reports on Progress in Physics 64: 815-884.

Orr SP, Metzger LJ, Pitman RK (2002). Psychophysiology of posttraumatic stress disorder. Psychiatr Clin North Am 25: 271-293.

Owens MJ, Nemeroff CB (1991). Physiology and pharmacology of corticotropin-releasing factor. Pharmacol Rev 43: 425-473.

Page ME, Abercrombie ED (1999). Discrete local application of corticotropin-releasing factor increases locus coeruleus discharge and extracellular norepinephrine in rat hippocampus. Synapse 33: 304-313.

Page ME, Berridge CW, Foote SL, Valentino RJ (1993). Corticotropin-releasing factor in the locus coeruleus mediates EEG activation associated with hypotensive stress. Neurosci Lett 164: 81-84.

Pieribone VA, Xu ZQ, Zhang X, Hokfelt T (1998). Electrophysiologic effects of galanin on neurons of the central nervous system. Ann N Y Acad Sci 863: 264-273.

Rutter JJ, Devilbiss DM, Waterhouse BD (2005). Effects of systemically administered cocaine on sensory responses to peri-threshold vibrissae stimulation: individual cells, ensemble activity, and animal behaviour. Eur J Neurosci 22: 3205-3216.

Schlifke I, Kuteeva E, Hokfelt T, Kokaia M (2006). Galanin expressed in the excitatory fibers attenuates synaptic strength and generalized seizures in the piriform cortex of mice. Exp Neurol 200: 398-406.

Schneidman E, Bialek W, Berry MJ (2003). Synergy, redundancy, and independence in population codes. J Neurosci 23: 11539-11553.

Shorten PR, Wall DJN (2001). A model of dispersion in perifusion systems. J Theoretical Med 3: 191-211.
Simons DJ (1978). Response properties of vibrissa units in rat SI somatosensory neocortex. J Neurophysiol 41: 798-820.

Simpson KL, Altman DW, Wang L, Kirifides ML, Lin RC, Waterhouse BD (1997). Lateralization and functional organization of the locus coeruleus projection to the trigeminal somatosensory pathway in rat. J Comp Neurol 385: 135-147.

Simpson KL, Waterhouse BD, Lin RC (1999). Origin, distribution, and morphology of galaninergic fibers in the rodent trigeminal system. J Comp Neurol 411: 524-534.

Smagin GN, Swiergiel AH, Dunn AJ (1995). Corticotropin-releasing factor administered into the locus coeruleus, but not the parabrachial nucleus, stimulates norepinephrine release in the prefrontal cortex. Brain Res Bull 36: 71-76.

Snyder K, Wang WW, Han R, McFadden K, Valentino RJ (2012). Corticotropin-releasing factor in the norepinephrine nucleus, locus coeruleus, facilitates behavioral flexibility. Neuropsychopharmacology 37: 520-530.

Swanson LW, Hartman BK (1975). The central adrenergic system. An immunofluorescence study of the location of cell bodies and their efferent connections in the rat utilizing dopamine-betahydroxylase as a marker. J Comp Neurol 163: 467-505.

Swerdlow NR, Geyer MA, Braff DL (2001). Neural circuit regulation of prepulse inhibition of startle in the rat: current knowledge and future challenges. Psychopharmacology (Berl) 156: 194-215.

Valentino RJ, Foote SL (1988). Corticotropin-releasing hormone increases tonic but not sensory-evoked activity of noradrenergic locus coeruleus neurons in unanesthetized rats. J Neurosci 8: 1016-1025.

Valentino RJ, Page ME, Curtis AL (1991). Activation of noradrenergic locus coeruleus neurons by hemodynamic stress is due to local release of corticotropin-releasing factor. Brain Res 555: 25-34.

Valentino RJ, Van Bockstaele E (2008). Convergent regulation of locus coeruleus activity as an adaptive response to stress. Eur J Pharmacol 583: 194-203.

Van Bockstaele EJ, Colago EE, Valentino RJ (1996). Corticotropinreleasing factor-containing axon terminals synapse onto catecholamine dendrites and may presynaptically modulate other afferents in the rostral pole of the nucleus locus coeruleus in the rat brain. J Comp Neurol 364: 523-534.

Van der Loos H (1976). Barreloids in mouse somatosensory thalamus. Neurosci Lett 2: 1-6.

Verhage M, McMahon HT, Ghijsen WE, Boomsma F, Scholten G, Wiegant VM et al (1991). Differential release of amino acids, neuropeptides, and catecholamines from isolated nerve terminals. Neuron 6: 517-524.

Waite PM (1973). The responses of cells in the rat thalamus to mechanical movements of the whiskers. J Physiol 228: 541-561.

Zhang SP, Bandler R, Carrive P (1990). Flight and immobility evoked by excitatory amino acid microinjection within distinct parts of the subtentorial midbrain periaqueductal gray of the cat. Brain Res 520: 73-82. 